\title{
Inventaire floristique et écologie des algues des rivières Kambo et Longmayagui de la zone humide de Douala (Cameroun)
}

\author{
Siegfried Didier DIBONG ${ }^{1,2,3}$ et Gildas Parfait NDJOUONDO ${ }^{1 *}$ \\ ${ }^{I}$ Département de Biologie des Organismes Végétaux, Faculté des Sciences, Université de Douala, \\ B.P. 24157 Douala, Cameroun. \\ ${ }^{2}$ Département des Sciences Pharmaceutiques, Faculté de Médecine et des Sciences Pharmaceutiques, \\ B.P. 2701 Douala, Cameroun. \\ ${ }^{3}$ Département d'Aquaculture, Institut des Sciences Halieutiques, B.P. 2701 Douala, Cameroun. \\ "Auteur correspondant; E-mail: parfaitgildas@yahoo.fr
}

\section{RESUME}

L'objectif général de l'étude est de contribuer à la connaissance des algues des rivières Kambo et Longmayagui de Douala. Les objectifs spécifiques ont consisté à mesurer les paramètres physico-chimiques des rivières, de faire les inventaires des algues et d'en dégager les variations spatio-temporelles en relation avec le milieu. La zone pélagique et les supports organiques ont été échantillonnés en amont, au milieu et en aval de chaque rivière. Au total, 105 espèces ont été identifiées, parmi lesquelles environ 40,59\% de Diatomées, 22,77\% de Cyanobactéries, 19\% d'Ulvophycées, 12,87\% de Zygophycées, 10,09\% de Chlorophycées, 7,9\% d'Euglénophycées et 1,9\% de Chrysophycées. Les Diatomées constituent la classe la plus fréquente dans les prélèvements des deux cours d'eau. Les genres Spirogyra et Navicula sont communes à toutes les stations. Les variations dans la composition des peuplements entre les rivières sont relativement faibles, avec des diversités plus importantes dans la rivière Kambo (92 espèces) et peu élevées dans la rivière Longmayagui (35 espèces).

() 2014 International Formulae Group. All rights reserved.

Mots clés : Phytoplancton, périphyton, diversité, rivières, Kambo, Longmayagui.

\section{INTRODUCTION}

La croissance démographique et le développement économique sont responsables de modifications majeures de l'eau. Cette pression sur l'environnement est tout particulièrement sensible en milieu tropical (Hatcher et al., 1989). La pollution est la modification et la dégradation d'un milieu aquatique, lié en général à un apport excessif de substances nutritives qui augmentent la production d'algues (Aurousseau, 2013). Les altérations du milieu sont la conséquence de facteurs anthropiques multiples (déversement des déchets urbains, agricoles et industriels). L'augmentation des apports en nutriments inorganiques et organiques dans les eaux influencent l'équilibre et la diversité des écosystèmes aquatiques notamment les lacs, les étangs et les cours d'eaux (Smetaceck et al., 1991). Les écosystèmes aquatiques continentaux hébergent des colonies d'algues sur tous les continents (Bartram et al., 1999). La relation entre la biodiversité et le fonctionnement des écosystèmes est une question écologique fondamentale. Il est indispensable de connaître les différents 
éléments abiotiques et biotiques en relation avec les algues qui composent les écosystèmes aquatiques et leur distribution dans le temps et dans l'espace notamment des rivières Kambo et Longmayagui (Bengtsson, 1998). Il apparaît difficile dans ce contexte de définir dans l'état actuel des connaissances les caractéristiques générales propres aux écosystèmes aquatiques de Douala.

L'objectif général de l'étude vise à déterminer la diversité et la distribution des algues des rivières Kambo et Longmayagui de Douala. Spécifiquement, il s'est agi de : (1) mesurer les facteurs physico-chimiques des deux rivières, (2) établir les inventaires des algues et (3) dégager les variations spatiotemporelles.

\section{MATERIEL ET METHODES \\ Sites d'étude}

La ville de Douala est subdivisée en 11 sous bassins versants (Meva'a et al., 2010). La région étudiée fait partie de la zone à climat équatorial côtier nord. La température moyenne annuelle est de $26,4{ }^{\circ} \mathrm{C}$. Les précipitations montrent que Douala est pluvieux avec des précipitations s'étendant sur 9 mois. Ces précipitations moyennes annuelles sont de 360,83 mm. L'humidité moyenne annuelle de l'air est de 78,3\%. L'évaporation moyenne annuelle est de $50,6 \%$. L'insolation moyenne annuelle est de $109,6 \%$. Le vent dominant est porteur de la mousson (Meva'a et al., 2010). L'étude s'est déroulée dans les rivières Kambo et Longmayagui où respectivement 4 et 3 stations d'échantillonnage ont été choisies selon le gradient longitudinal du cours d'eau. La rivière Kambo couvre le quartier Yassa, stations 1 et 2 situées en amont et est exposée aux pollutions agropastorales. La station 1 est isolée de la station 2 par un remblaie de terre. Les quartiers Arir, station 3 (milieu) et Cogefar, station 4 (aval) sont exposés aux pollutions par les déchets ménagers. La rivière Longmayagui (amont) couvre le quartier Logbaba et est exposée aux pollutions de la zone industrielle de Bassa notamment la Société Anonyme des Brasseries du Cameroun (SABC). Les quartiers Diboum et Cité des Billes (milieu), et Bobongo (aval) sont exposés aux pollutions par les déchets ménagers et les activités agropastorales.

\section{Période d'échantillonnage}

L'échantillonnage s'est déroulé du 15 décembre 2013 au 15 mars 2014. Quatre campagnes d'échantillonnage ont été effectuées respectivement le 15 décembre 2013, le 15 janvier, le 15 février et le 15 mars 2014.

\section{Paramètres physico-chimiques}

Les paramètres physico-chimiques ont été mesurés entre $7 \mathrm{~h}$ et $11 \mathrm{~h}$ du matin aux différents sites de prélèvement. L'oxygène dissout a été mesuré par un oxymètre WTW. Les températures ambiantes et des solutions, la conductivité, les taux de solide dissous, la salinité et le $\mathrm{pH}$ de l'eau ont été mesurés par un multiparamètre $\mathrm{pH} / \mathrm{TDS} / \mathrm{COND} / \mathrm{Salt} / \mathrm{T}$ de marque OAKTON. La transparence de l'eau a été mesurée par un disque de Secchi. Le débit de l'eau a été mesuré à l'aide d'une corde munie d'un flotteur et d'un chronomètre. Ces paramètres ont été mesurés dans chaque site pour chaque campagne d'échantillonnage. Après collecte, les échantillons d'eau ont été stockés dans des bocaux $(1000 \mathrm{ml})$ et conservés au frais et à l'obscurité dans une glacière pour l'analyse du nitrate au laboratoire d'hydrobiologie de l'Université de Yaoundé. Les nitrates ont été dosés par spectrophotométrie après ajout du nitraver $\mathrm{V}$.

\section{Echantillonnage qualitatif}

Les milieux échantillonnés sont des eaux courantes excepté la station 1 , qui est une eau marnante. Le filet à plancton a été utilisé pour la filtration des algues planctoniques. Pour cela, il a été lancé dans l'eau et tiré suivant une distance de $10 \mathrm{~m}$ dans la zone euphotique. Le périphyton a été prélevé par expression des végétaux immergés. Le contenu du filet a été déversé dans un pilulier étiqueté suivant les stations. Le contenu issu de l'expression des végétaux immergés a été déversé dans un pilulier étiqueté suivant les stations. Le formol à $40 \%$ a été utilisé pour la fixation des échantillons à $5 \%$ du volume du pilulier. Ces échantillons ont été ensuite conservés à l'obscurité dans une glacière. 


\section{Echantillonnage quantitatif}

Dans chaque station un bocal de 1000 $\mathrm{ml}$ a été utilisé pour le prélèvement de $500 \mathrm{ml}$ d'eau fixées ensuite à $5 \%$ du volume par le formol concentré à $40 \%$ et étiqueté selon les stations. Un bocal a été utilisé pour le périphyton pressé selon une surface de $(30 \times$ 30) $\mathrm{cm}^{2}$ et dilué à de l'eau distillée jusqu'à $300 \mathrm{ml}$ puis fixé au formol à 5\% du volume. Chaque bocal a été étiqueté. Ces échantillons ont été introduits dans la glacière pour la conservation à l'obscurité.

\section{Analyse qualitative}

Au laboratoire, des sous échantillons de $10 \mathrm{ml}$ ont été prélevés. Après sédimentation des individus au fond des béchers utilisés pour le sous échantillonnage pendant $24 \mathrm{~h}$, une goutte de chaque échantillon a été montée entre lame et lamelle par un compte goutte puis observée au microscope photonique droit. Dix préparations ont été effectuées pour chaque échantillon. Les identifications ont été effectuées directement au microscope à partir des transects linéaires de la gauche vers la droite de la lame. Mais des dessins et des photographies ont été effectués pour les individus difficiles à identifier pour plus de détails concernant les mensurations. Les clés d'identification suivantes ont été utilisées : Iltis (1980), Bourrelly (1966, 1968, 1970), Compère (1967, 1974, 1976), Berne (2007), Grönblad et al. (1958), Krammer et LangeBertalot (2000).

\section{Analyse quantitative}

Après dépôt des bocaux échantillonnés, une homogénéisation a été effectuée. Des sous échantillons de $10 \mathrm{ml}$ par bécher ont été effectués. Après agitation de chaque bécher laissé au repos et à l'obscurité pendant 24 h, 1 $\mathrm{ml}$ du contenu du fond a été prélevé par une micropipette puis versé dans la lame de comptage de Manassé. Le microscope photonique de marque OLYMPUS a été utilisé pour le comptage des individus. 400 individus au moins ont été comptés. L'unité de comptage des filaments a été fixée à 100 $\mu \mathrm{m}$ comme 1 individu. Les colonies et les coenobes ont été considérées comme 1 individu (Iltis, 1980).
Calcul des paramètres: diversité des taxons observés

La richesse spécifique

C'est le nombre total des diverses catégories taxonomiques auxquelles appartiennent les organismes prélevés à une station d'échantillonnage. Elle mesure la diversité la plus élémentaire, fondée directement sur le nombre total d'espèces dans un site (cette méthode dépend de la taille des échantillons et ne considère pas l'abondance relative des différentes espèces) (Travers, 1964).

L'indice de diversité de Shannon-Weaver et l'équitabilité de Piélou

L'indice de diversité de Shannon-Weaver est calculé de manière suivant :

$\mathrm{H}^{\prime}=-\sum\left((\mathrm{ni} / \mathrm{N}) \times \log _{2}(\mathrm{ni} / \mathrm{N})\right)\left(\right.$ avec $\mathrm{H}^{\prime}=$ indice de diversité de Shannon, ni $=$ nombre d'individus appartenant à une espèce, $\mathrm{N}=$ nombre total d'espèces) ;

L'équitabilité est donnée par la formule suivante :

$E^{\prime}=H^{\prime} / \log _{2} S$ (avec E' = indice d'équitabilité, $\mathrm{H}=$ indice de Shannon, $\mathrm{S}=$ volume total de l'échantillon sédimenté).

La comparaison entre la flore de deux stations données a été faite par le coefficient de similartité de Sorensen

Similarité de Sorensen : $\mathrm{C}_{\mathrm{s}}=\frac{2 j}{2 j+a+b}$

Soit $\mathrm{A}$ et $\mathrm{B}$ deux milieux, $\mathrm{a}=$ nombre d'espèces présentes dans le milieu $\mathrm{A}, \mathrm{b}=$ nombre d'espèces présentes dans le milieu $B$ et $\mathrm{j}=$ nombre d'espèces communes aux 2 milieux; l'indice de similarité de Sorensen varie de 0 (absence de similarité) à 1 (milieux identiques).

\section{Densité}

Le calcul de la densité s'exprime par la formule de Groga (2012) : $\mathrm{D}=\mathrm{Ni} \times \mathrm{R} \times$ $1000 / \mathrm{v}$ (avec $\mathrm{D}=$ densité en nombre d'individus par litre, $\mathrm{R}=$ rapport entre la surface de la cellule de comptage et la surface du champ oculaire), $1000=$ facteur de conversion en litre, $\mathrm{v}=$ volume d'échantillon sédimenté en $\mathrm{ml}$ et $\mathrm{Ni}=$ moyenne du nombre d'individus d'une espèce. 


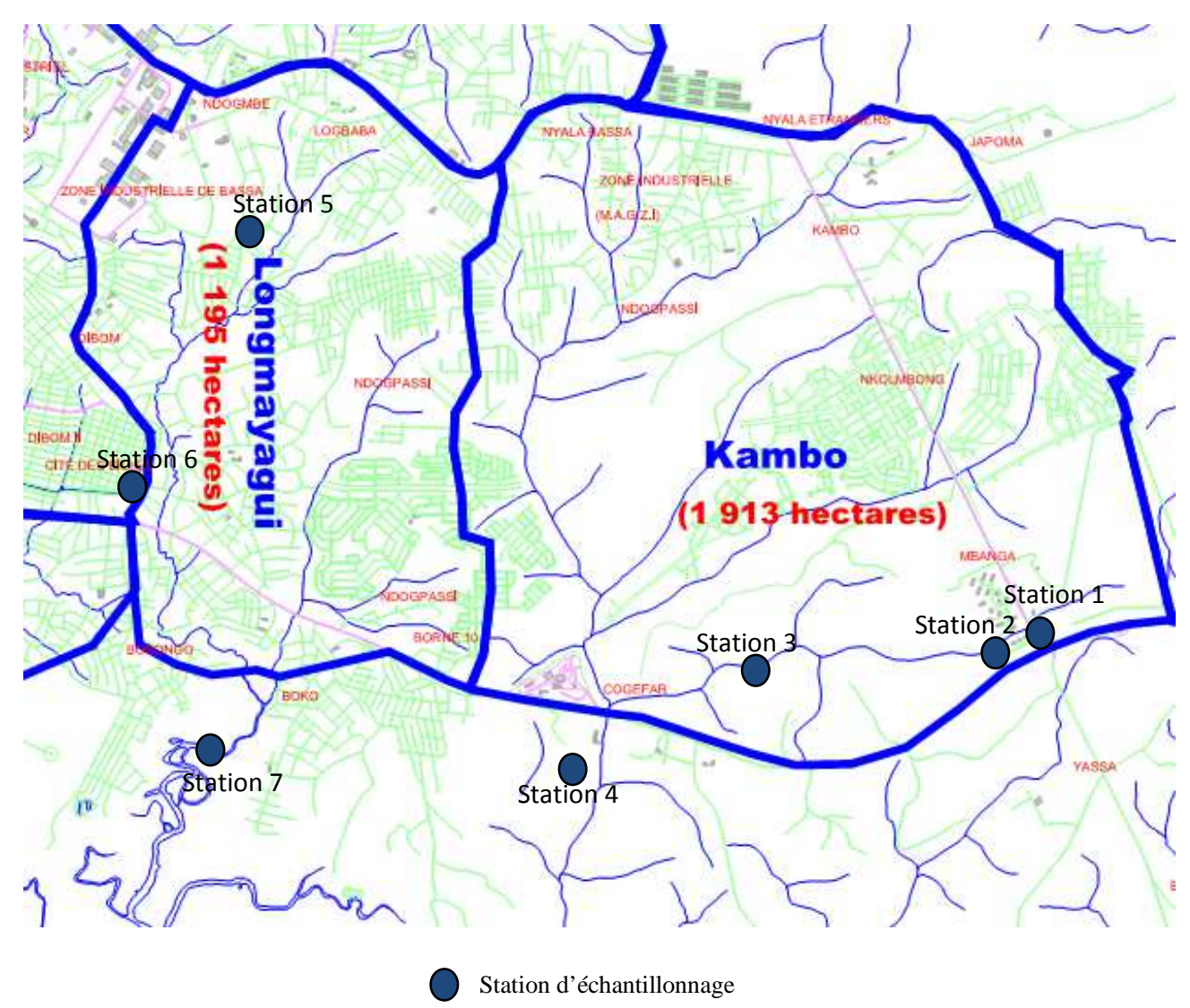

Figure 1 : Carte des rivières montrant les stations d'échantillonnage.

\section{Analyses statistiques}

Une analyse en Composantes Principales (ACP) a été utilisée pour ordonner les stations en fonction des paramètres abiotiques. L'Analyse Factorielle des Correspondances (AFC) a été appliquée à la composition du peuplement afin de regrouper les stations d'échantillonnage en fonction de leurs similarités floristiques. Ces analyses ont été effectuées avec le logiciel $\mathrm{R}$ version $\mathrm{R}$ i 386 3.0.1 pour la construction des ACP et XLSTAT 2014 pour la construction des AFC et des dendogrammes.

\section{RESULTATS}

\section{Paramètres physico-chimiques}

La température ambiante varie de 28,32 $\pm 1,07{ }^{\circ} \mathrm{C}$ (station 6) à 29,80 $\pm 0,25{ }^{\circ} \mathrm{C}$ (station 1). La température de l'eau varie de $27,10 \pm 0,44$ (station 4) à 28,60 $\pm 0,20$ (station
1). La transparence varie de 37,00 $\pm 5,00$ (station 6) à 62,00 $\pm 3,00$ (station 4). Les stations 2 et 3 n'ont pas de transparence. La profondeur de l'eau varie de 50,00 $\pm 0,00$ (station 2) à 150,00 $\pm 0,00$ (station 4). Le débit de l'eau varie de $0 \mathrm{~m} / \mathrm{s} \pm 0,00$ (station 1 ) à $0,20 \pm 0,02 \mathrm{~m} / \mathrm{s}$ (station 2). Le taux de solides dissous varie de 34,60 $\pm 0,99 \mathrm{ppm}$ (station 3) à 185,00 $\pm 9,00 \mathrm{ppm}$ (station 7). La conductivité de l'eau varie de 66,71 $\pm 2,61$ $\mu \mathrm{S} / \mathrm{cm}$ (station 4) à $258,00 \pm 50,00 \mu \mathrm{S} / \mathrm{cm}$ (station 7). La salinité de l'eau varie de 38,30 $\pm 0,65 \mathrm{ppm}$ (station 2) à $150,00 \pm 8,00 \mathrm{ppm}$ (station 5). Le pH de l'eau varie de 6,43 \pm 0,16 (station 2) à 7,12 $\pm 0,11$ (station 6). Le taux d'oxygène dissous varie de 5,27 $\pm 0,02$ $\mathrm{mg} / \mathrm{l}$ (station 2) à 6,67 $\pm 0,01 \mathrm{mg} / \mathrm{l}$ (station 7). Les nitrates varient de $0,71 \pm 0,42$ (station 2) à $2,97 \pm 0,08$ (station 7) (Tableau 1). 


\section{Typologie des stations}

Les résultats de l'ACP montrent l'organisation des 10 variables mesurés sur les plans F1 x F2, représentés sous forme de cercles de corrélations (Figure 2). L'axe F1 est corrélé positivement au $\mathrm{pH}$, à la transparence, à la conductivité, au taux de solides dissouts et à la salinité. Il rapproche les stations 5, 6 et 7 . Le côté négatif n'est corrélé à aucune variable et rapproche les stations 2 et 3 . L'axe F2 est corrélé positivement aux températures ambiantes et de l'eau, et rapproche la station 1 . Il est corrélé négativement à l'oxygène dissout, à la profondeur de l'eau et au débit. Cet axe rapproche la station 4 .

La composante principale F1 définit donc un gradient de pollution organiques et en sels (Figure 2).

La classification ascendante hiérarchique a permis d'individualiser 3 groupes de stations définis par leurs caractéristiques physico-chimiques mesurées près coupure du dendrogramme à $\mathrm{x}$ de dissimilarité.

- Le groupe I : formé des stations 1, 2 et 3 soumises aux faibles valeurs de transparence, de $\mathrm{pH}$, de salinité et de conductivité ;

- Le groupe II : formé des stations 5, 6 et 7 soumises aux valeurs élevées de transparence, de $\mathrm{pH}$, de salinité et de conductivité.

Ces résultats permettent donc de distinguer suivant le gradient de pollution des stations du site 1 (Kambo) des stations du site 2 (Longmayagui). Ceci permet de montrer que le site 1 est moins pollué que le site 2, pollué. La station 4 se démarque des autres stations du site 1 (Figure 3 ).

\section{Richesse spécifique}

La richesse spécifique est élevée, au total 105 espèces réparties en 4 embranchements, 8 classes, 36 familles et 59 genres ont été recensées. La classe la plus représentée est celle des Bacillariophycées avec 41 espèces $(40,59 \%)$. La classe la moins représentée est celle des Chrysophycées avec une espèce $(1,9 \%)$ (Figure 4$)$.

$\mathrm{Au}$ site 1 (Kambo) la richesse spécifique est de 92 espèces réparties en 4 embranchements, 8 classes et 56 genres. Au site 2 (Longmayagui), la richesse spécifique est de 35 espèces. Elles sont réparties en 4 embranchements, 5 classes et 25 genres.

\section{Indice de diversité}

$\mathrm{Au}$ site 1, l'indice de diversité de Shannon-Weaver est élevé et varie de 4,95 bits (station 2) à 3,89 (station 3). L'indice d'équitabilité de Piélou varie de $72 \%$ (station 3) à 95,78\% (station 1) (Figures 6a, 7a). Au site 2, l'indice de diversité de ShannonWeaver varie de 4,62 (station 5) à 4,28 (station 6). La régularité varie de 91,05\% (station 5) à 84,35\% (station 6) (Figures 6b, 7b).

\section{Indice de similitude de Sorensen}

$\mathrm{Au}$ site 1 l'indice de similitude est élevé entre les stations 2 et $3(58,82 \%)$ avec 30 espèces communes. Cet indice de similitude est faible entre les stations 1 et 2 $(12,82 \%)$ avec 10 espèces communes (Figure 8a). Le nombre d'espèces communes aux quatre stations est de 13. Au site 2 (Longmayagui), 35 espèces ont été recensées : 34 espèces aux stations 5 et 6 , et 35 espèces à la station 7. L'indice de similitude est élevé $(90,41 \%)$ entre les stations 5 et 6 , et les stations 5 et 7 avec respectivement 33 espèces communes. Il est minimal $(88,23 \%)$ entre les stations 6 et 7 avec 30 espèces communes. Le nombre d'espèces communes aux trois stations est de 34 (Figure 8b).

\section{Variation spatio-temporelle des stations en fonction des individus}

Les résultats de l'AFC montrent 4 groupes des stations définis par des espèces qui leurs sont caractéristiques. L'axe 1 (40,59\% d'inertie) corrélé positivement au groupe II formé des stations 5 , 6 et 7 est caractérisé par les espèces Achnanthes minutissima var. saprophia, Cyclotella sp., 
Mastogloia smithii, Melosira sp. 2, Melosira varians, Navicula nivalis, Nitzschia sigma, Nitzschia sp., Stephanodiscus hantzschii, Ankistrodesmus gracilis, Cladophora holsatica, Cosmarium subtumidum, Eremosphaeria sp., Gonatozygon monotaenium, Gonatozygon sp. 1, Gonatozygon sp. 2, Kirchneriella irregularis, Monoraphidium concortum, Oedogonium sp., Uronema elongatum, Aphanizomenon flosaquae, Aphanocapsa litoralis, Oscillatoria sp., Pseudanabaena catenata, Phacus orbicularis. L'axe $2(23,87 \%$ d'inertie) est corrélé positivement au groupe I formé des stations 1,2 et 3 caractérisé par les espèces Amphipleura pellucida, Anomoeoneis sphaerophora, Aulacoseira granulata, Caloneis bacillum, Cymatopleura solea, Cymbella amphicephata, Cymbella cesatii, Cymbella naviculiformis, Diatoma mesodon, Diatoma sp., Melosira sp.1, Meridion circulare, Nitzschia fonticola, Nitzschia sigma, Pinnularia gibba, Stephanodiscus minutulus, Closterium parvulum, Enteromorpha flexuosa, Ulothrix sp., Uronema sp., Lymbya sp., Nostoc sp. 2, Oscillatoria boryana, Oscillatoria cryptovaginata, Raphidiopsis curvata, Stichococcus ampulliformis, Trachelomonas hispida. L'axe 2 est corrélé négativement au groupe III formé de la station 4, caractérisée par les espèces Achnanthes minutissima var scotica, Diatoma tenuis, Navicula accomoda, Navicula Cryptotenelloides, Closterium ehrenbergii, Aphanocapsa holsatica, Binucleria eriensis, Rivularia sp., Tabellaria flocculosa, Calothrix sp., Synechococcus aeruginosus, Pleurosigma directum. Les axes 1 et 2 sont corrélés positivement et négativement au groupe IV qui est celui des espèces communes (Figure 9).

Le rapprochement des stations en fonction des facteurs abiotiques et des stations en fonction des espèces montre que les espèces des stations 1,2 et 3 formant le groupe I sont soumises aux eaux non polluées alors que les espèces des stations 5,6 et 7 formant le groupe II sont soumises aux eaux polluées. Les espèces de la station 4 malgré ses valeurs des facteurs abiotiques proches des autres stations du site 1 n'est pas proche d'autres stations, ce qui laisse penser que la station 4 est un milieu moins pollué.

\section{Densité des algues}

$\mathrm{Au}$ site 1, la densité la plus élevée est observée à la station 1 et représentée par l'embranchement des Chlorophyta (45,45\%). Les stations 2 et 3 sont à dominance des Chromophyta $\quad(36,85 \%$ et $\quad 44,91 \%$ respectivement) suivie des Cyanophyta $(28,45 \%$ et $25,14 \%$ respectivement). A la station 4 les Chromophyta restent élevées $(34,52 \%)$ suivie des Cyanophyta (27,38\%). Les Euglenophyta dans les stations et la densité la plus faible s'observe à la station 1 $(2,27 \%)$. Au site 2, les Chromophyta sont majoritaires avec le pourcentage le plus élevé observé à la station $5(60,06 \%)$, suivis des Chlorophyta dont la densité la plus élevée est relevée à la station $7(39,17 \%)$ (Figure 10).

Les Diatomées sont plus représentées dans le site 1 que dans le site 2 . Les espèces qui colonisent le site sont de petites tailles et appartiennent pour la plupart aux Diatomées pennées. Ces espèces sont dominées par le périphiton. La spirogyre a été retrouvée dans tous les milieux et dans toutes les stations.

Les Euglénophycées sont plus importantes au site $2(9,8 \%)$ qu'au site 1 $(7,7 \%)$. Les Cyanophycées sont plus importantes au site $1(25,55 \%)$ qu'au site 2 (9,8\%). Les Zygophycées, Ulvophycées et Chrysophycées sont absentes au site 2. Les Charophycées sont plus abondantes au site 1 $(2,2 \%)$ qu'au site $2 \quad(2,1 \%)$. Les Bacillariophycées sont plus importantes au site $2(51,51 \%)$ qu'au site $1(41,11 \%)$ (Figure $5)$. 
Tableau 1: Paramètres physico-chimiques des différentes stations.

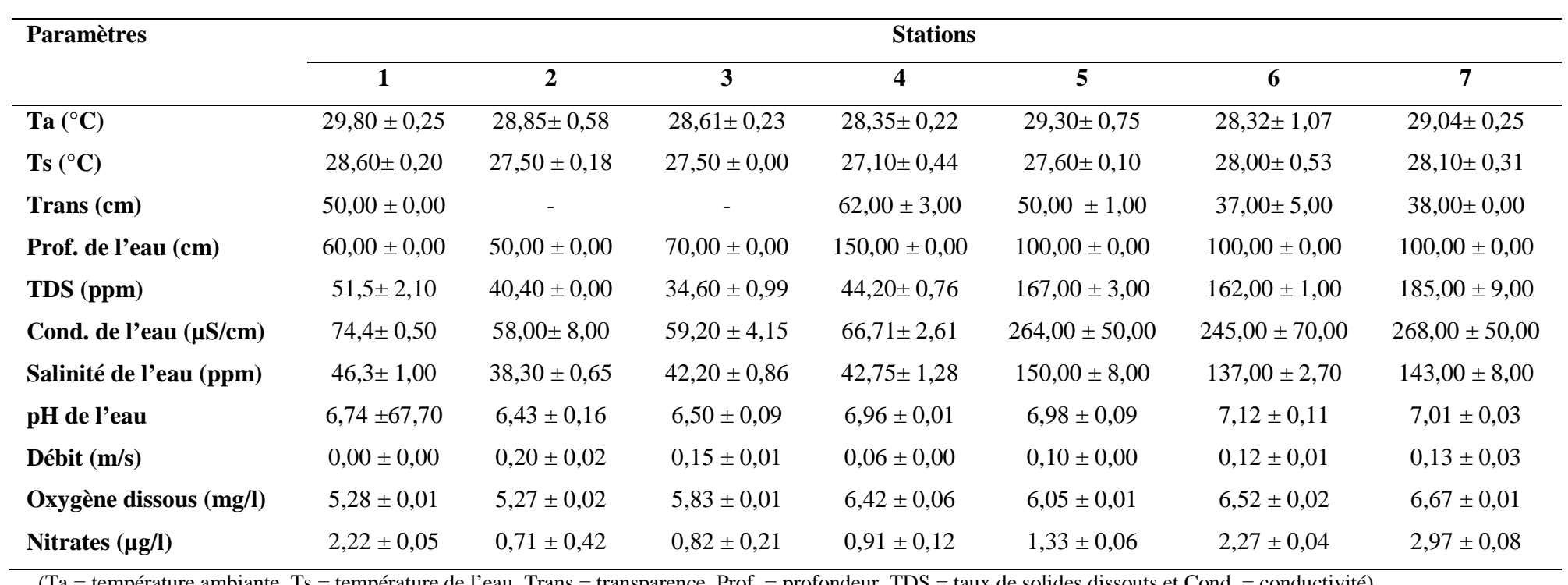

$(\mathrm{Ta}=$ température ambiante, $\mathrm{Ts}=$ température de l'eau, Trans = transparence, Prof. = profondeur, TDS = taux de solides dissouts et Cond. = conductivité $)$ 


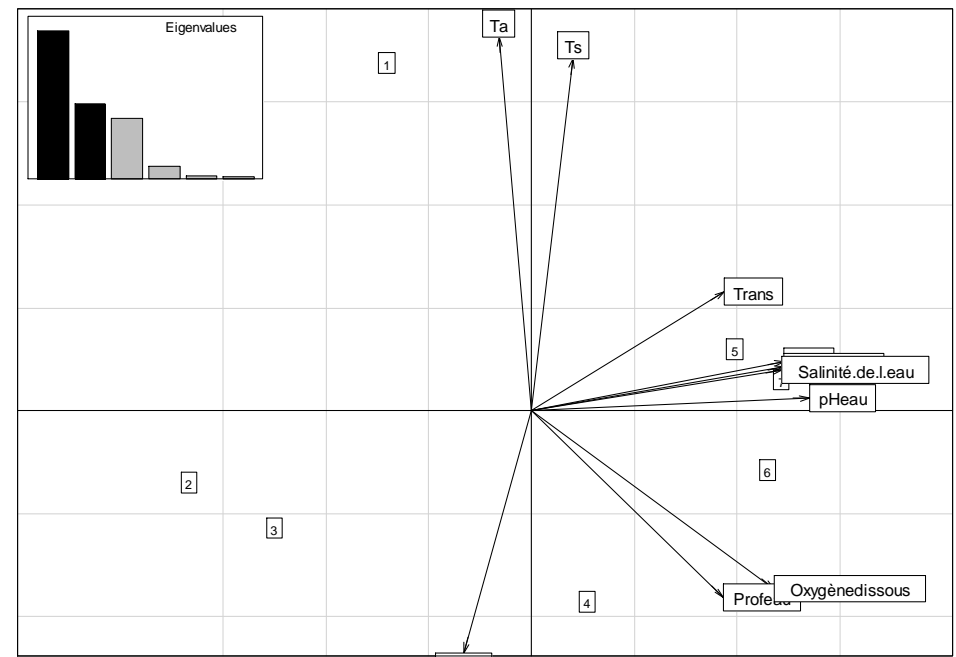

Figure 2 : Représentation des 10 paramètres physico-chimiques dans le plan factoriel 1-2 de l'ACP. $(\mathrm{Ta}=$ température ambiante, $\mathrm{Ts}=$ température de l'eau, Trans. $=$ transparence, Prof. $=$ profondeur , Cond.$=$ conductivité et TDS $=$ taux de solides dissouts).

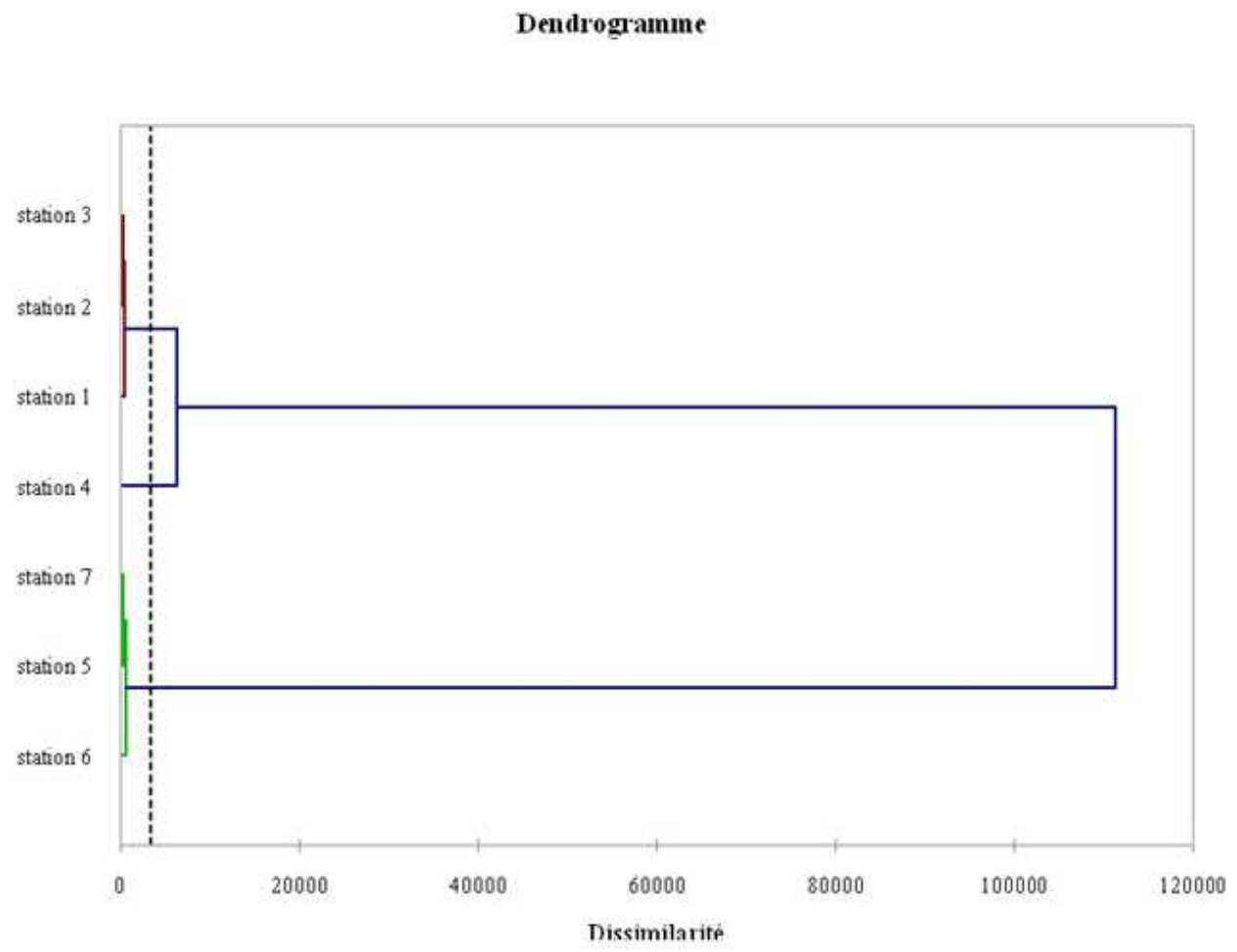

Figure 3 : Dendogramme illustrant le rapprochement des 7 stations en fonction des facteurs abiotiques dans les rivières Kambo et Longmayagui. 


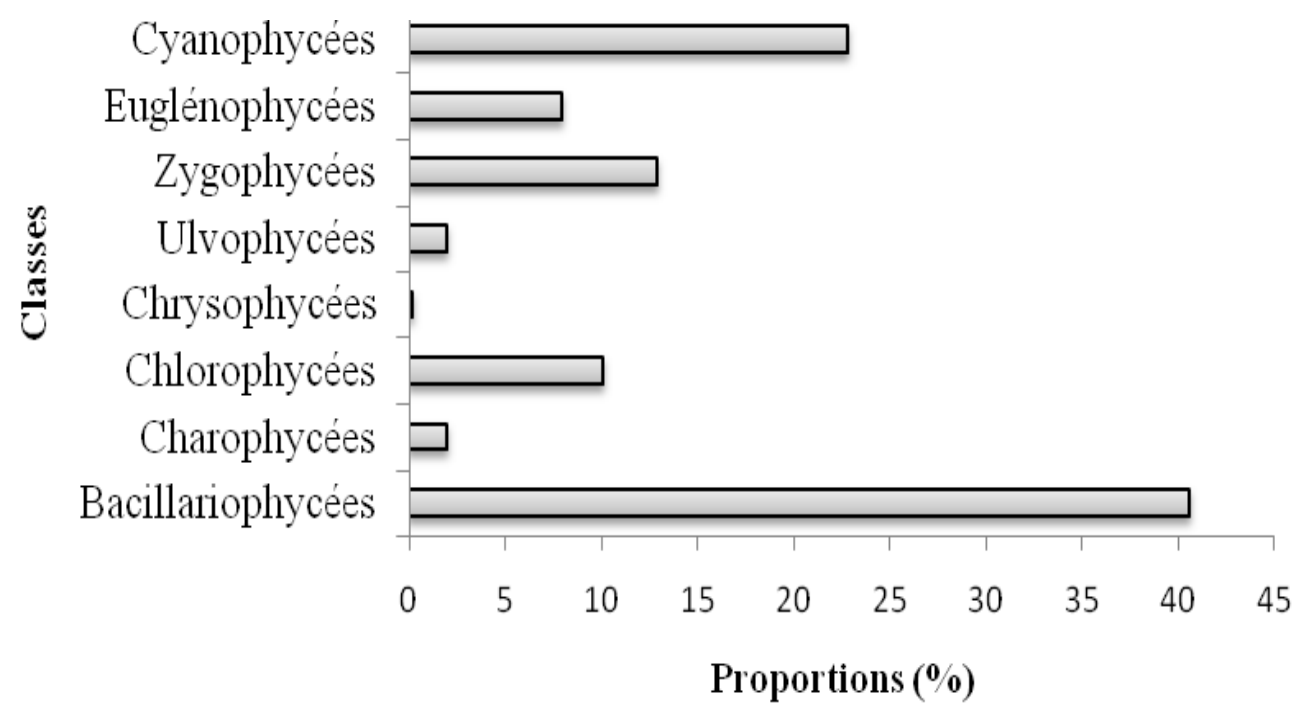

口Richesse spécifique

Figure 4 : Proportions du nombre d'espèces dans les différentes classes d'algues de l'ensemble des stations étudiées.

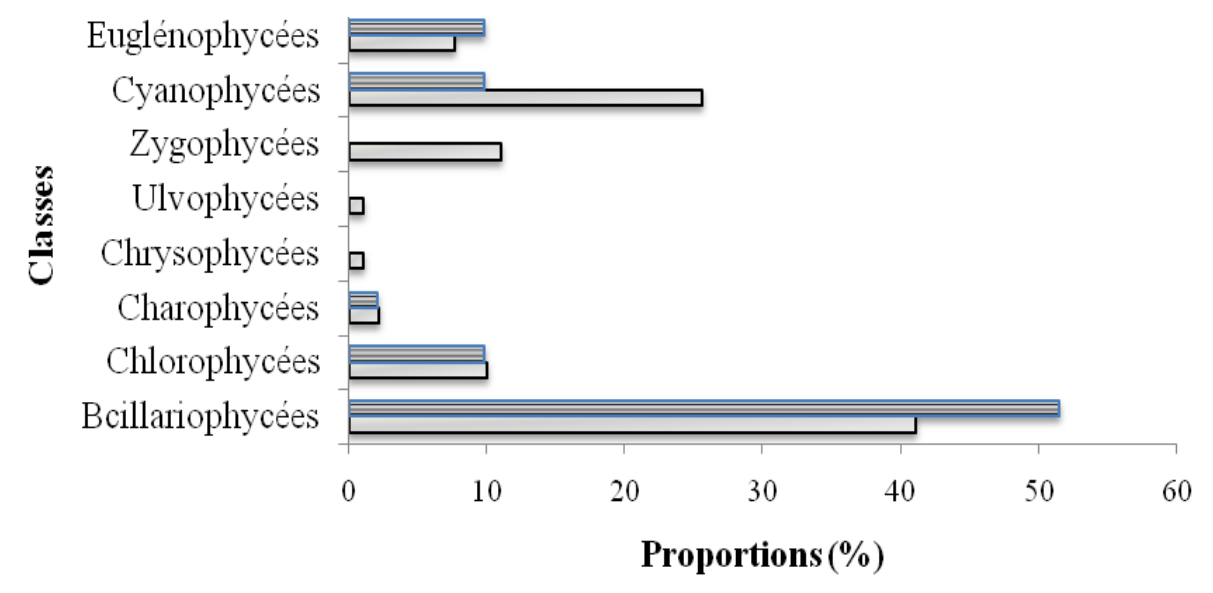

目site 2 口site 1

Figure 5: Proportions du nombre d'espèces par classes d'algues dans les sites de Kambo et Longmayagui. 


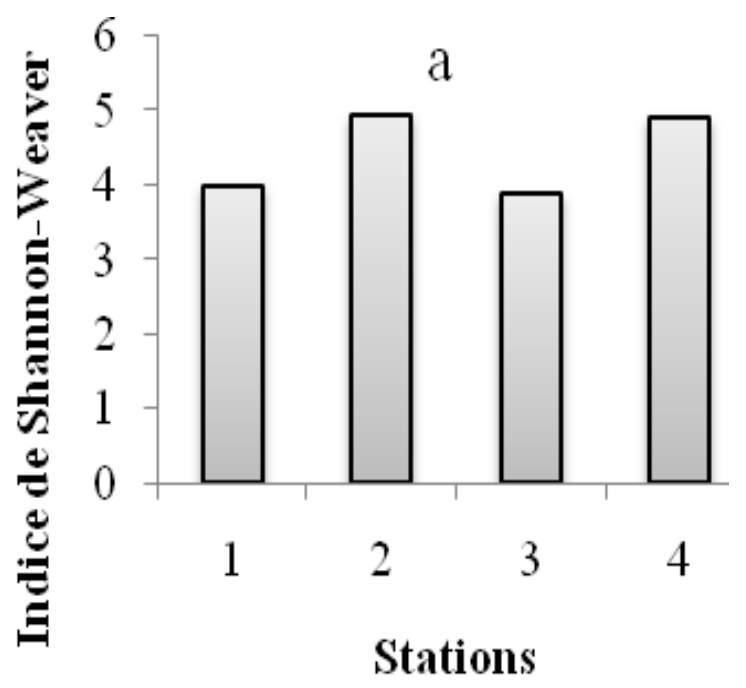

口Indice de Shannon-Weaver

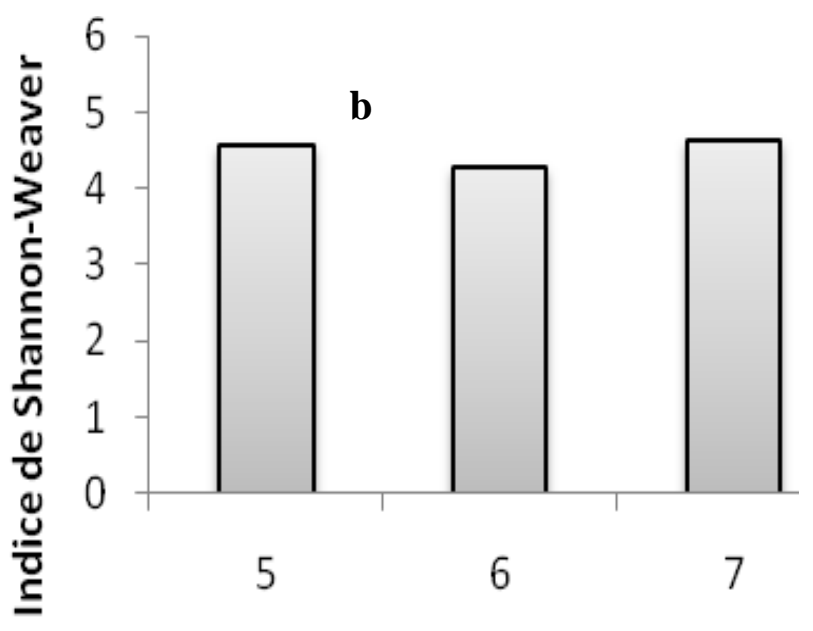

Stations

QIndice de Shannon-Weaver

Figure 6 : Indice de diversité de Shannon-Weaver dans les sites 1 (a) et 2 (b). 


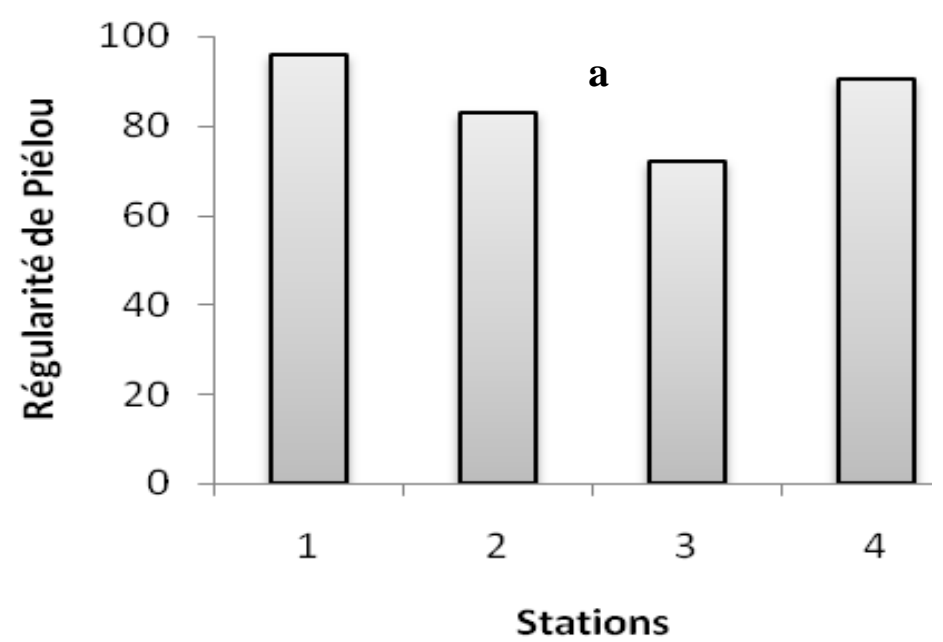

$\square$ Régularité de Piélou

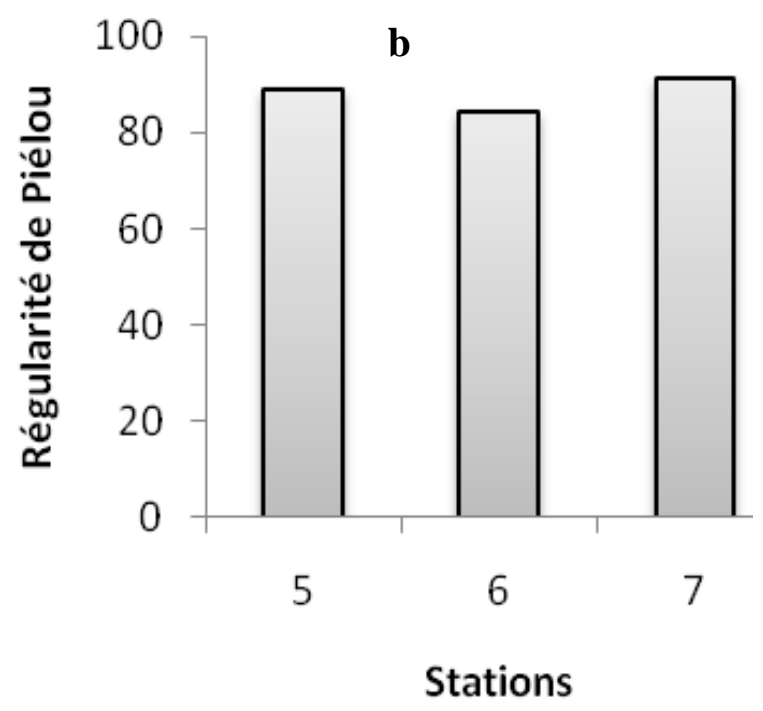

$\square$ Régularité de Piélou

Figure 7 : Régularité de Piélou dans les sites 1 (a) et 2 (b). 

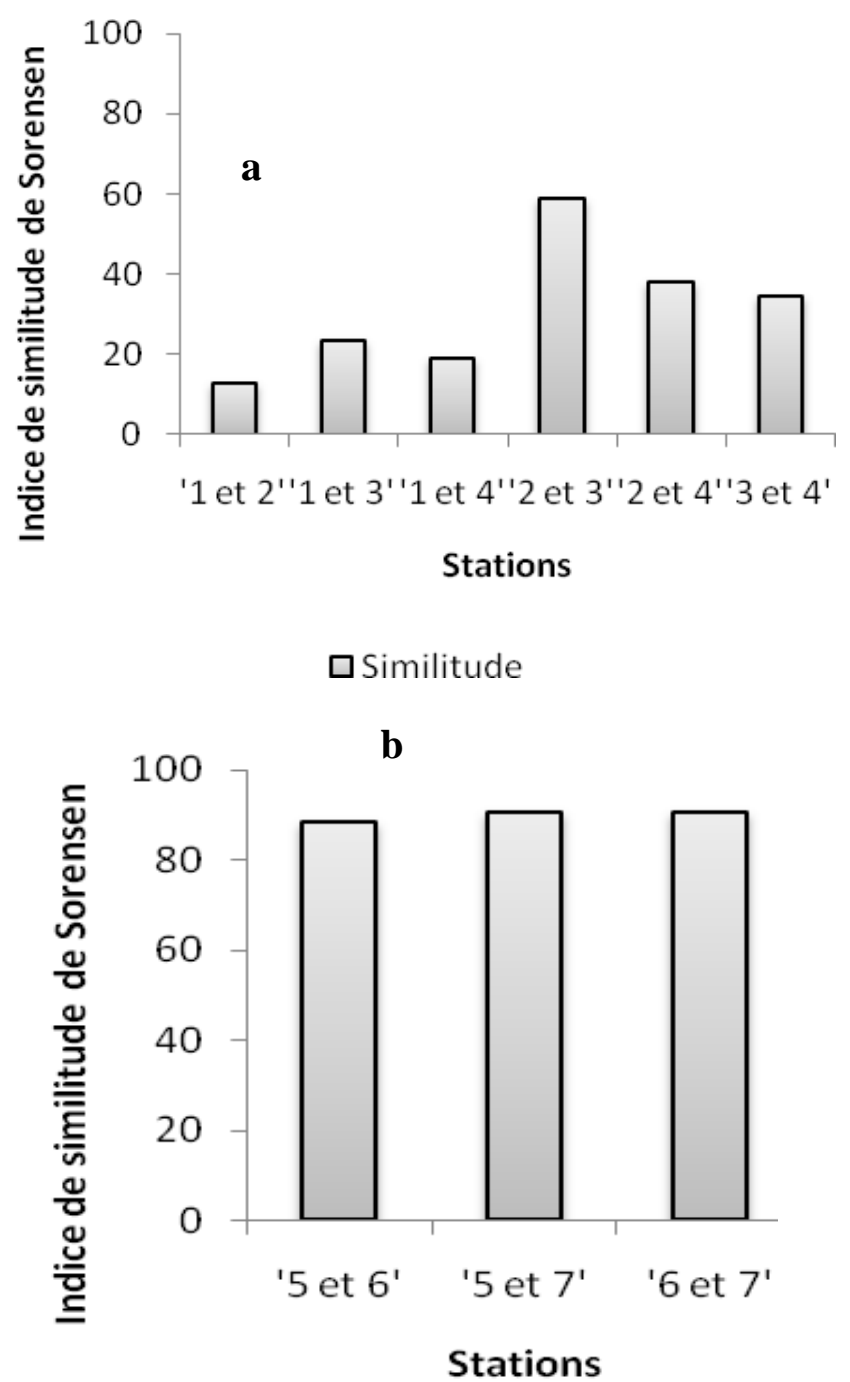

$\square$ Similitude

Figure 8 : Indice de similitude de Sorensen dans les sites 1 (a) et 2 (b). 


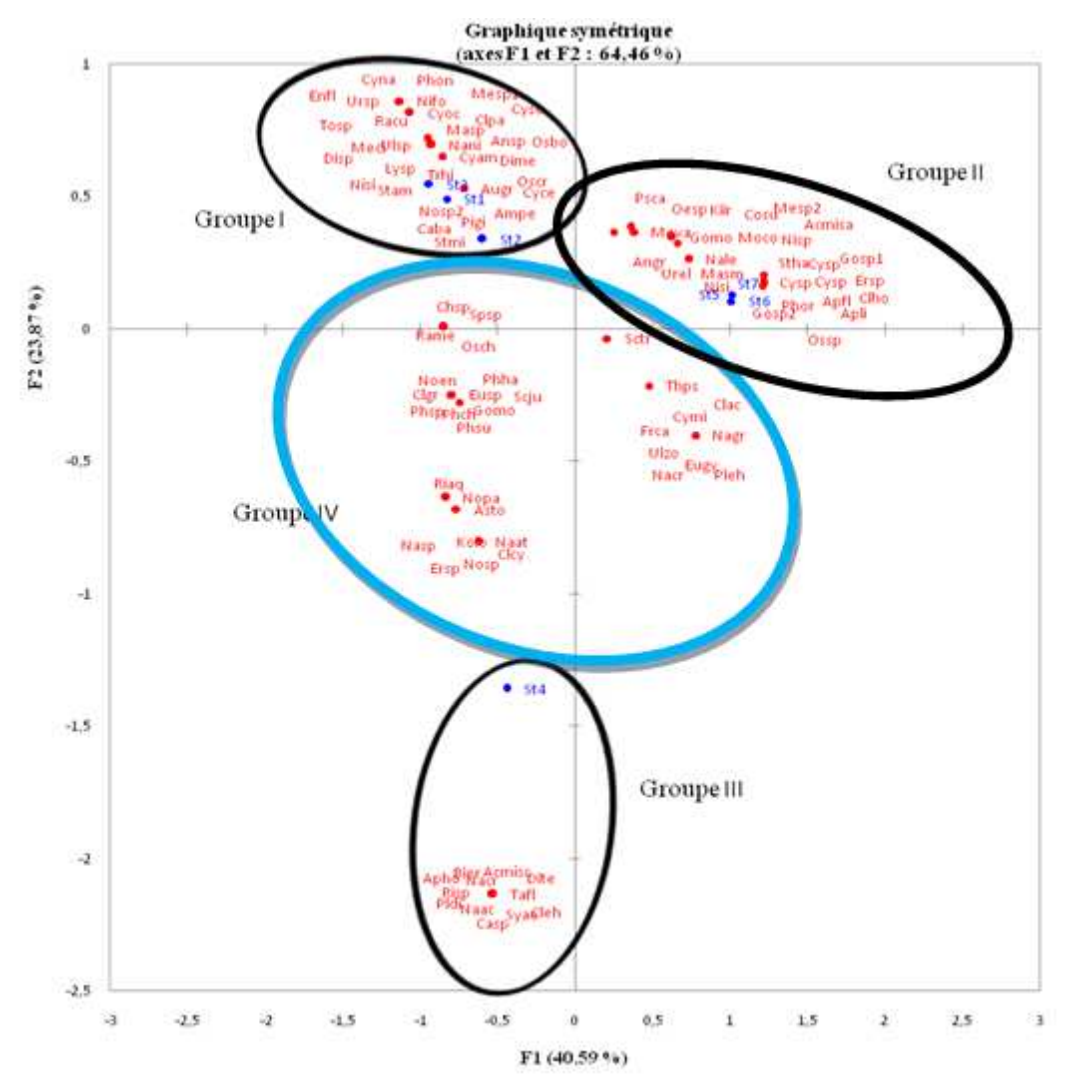

Figure 9 : Représentations des groupes de l'AFC sur le plan factoriel 1-2 de l'AFC. Les 7 stations sont représentées en bleue et les $\mathrm{x}$ espèces en rouge. Acmisa = Achnanthes minutissima var saprophia, Acmisc = Achnanthes minutissima var scotica, Ampe = Amphipleura pellucida, Ansp = Anomoeoneis sphaerophora, Augr $=$ Aulacoseira granulata, $\mathrm{Caba}=$ Caloneis bacillum, Cyoc $=$ Cyclotella ocellata, Cysp = Cyclotella sp., Cyso = Cymatopleura solea, Cyam $=$ Cymbella amphicephata, Cyce $=$ Cymbella cesatii, Cymi $=$ Cymbella microcephala, Cyna $=$ Cymbella naviculiformis, Dime $=$ Diatoma mesodon, Disp $=$ Diatoma sp., Dite $=$ Diatoma tenuis, Frca $=$ Fragilaria capucina, Gosp 1 = Gomphonema sp. 1, Gosp $2=$ Gomphonema sp. 2, Masm $=$ Mastogloia smithii, Mesp $1=$ Melosira sp. 1, Mesp $2=$ Melosira sp. 2, Meva = Melosira varians, Meci =Meridion circulare, Naac $=$ Navicula accomoda, Naat $=$ Navicula atomus, Nagr $=$ Navicula gregana, Nacr $=$ Navicula Cryptotenelloides, Nale $=$ Navicula lenzii, Nani $=$ Navicula nivalis, Nasp $=$ Navicula sp., Nifo $=$ Nitzschia fonticola, Nisi $=$ Nitzschia sigma, Nisp $=$ Nitzschia sp., Pigi $=$ Pinnularia gibba, Pldi $=$ Pleurosigma directum, Stmi $=$ Stephanodiscus minutulus, Stha $=$ Stephanodiscus hantzschii, Tafl $=$ Tabellaria flocculosa, Thps $=$ Thalassiosira pseudonana, Angr $=$ Ankistrodesmus gracilis, Bier $=$ Binucleria eriensis, Chsp $=$ Chara $\mathrm{sp} .$, Clho $=$ Cladophora holsatica, Clac $=$ Closterium acerosium, Clcy $=$ Closterium cynthia, Cleh $=$ Closterium ehrenbergii, Clgr $=$ Closterium gracile, Clle $=$ Closterium leibleinii, Clpa $=$ Closterium parvulum, Core $=$ Cosmarium rectangulare, Cosu $=$ Cosmarium subtumidum, Enfl = Enteromorpha flexuosa, Ersp $=$ Eremosphaeria sp., Gomo $=$ Gonatozygon monotaenium, Gosp $=$ Gonatozygon sp., Kiir $=$ Kirchneriella irregularis, Kolo $=$ Koliella longiseta, Masp =Mallomonas sp., Moco $=$ Monoraphidium concortum, Oesp $=$ Oedogonium sp., Pleh= Pleurotaenium ehrenbergii, Spsp $=$ Spirogyra sp., Tosp $=$ Tolypothrix sp., Ulzo = Ulothrix zonata, Ulsp = Ulothrix sp., Urel = Uronema elongatum, Ursp = Uronema sp., Apfl $=$ Aphanizomenon flos-aquae, Apho = Aphanocapsa holsatica, Apli = Aphanocapsa litoralis, Casp $=$ Calothrix sp., Cysp $=$ Cylindrospermum sp., Lysp = Lymbya sp., Mila $=$ Microcoleus lacustris, Noen $=$ Nostoc entophyllum, Nopa $=$ Nostoc paludosum, Nosp $1=$ Nostoc $\mathrm{sp} .1$, Nosp $2=$ Nostoc $\mathrm{sp} .2$, Osbo $=$ Oscillatoria boryana, Osch = Oscillatoria chalybea, Ossp $=$ Oscillatoria sp., Oscr $=$ Oscillatoria cryptovaginata, $\mathrm{Phch}=$ Phormidium chlorinum, $\mathrm{Phha}=$ Phormidium hamelii, Phsu $=$ Phormidium subfuscum, Psca $=$ Pseudanabaena catenata, Racu $=$ Raphidiopsis curvata, Rame $=$ Raphidiopsis mediterranea, Riaq $=$ Rivularia aquatica, Risp $=$ Rivularia sp., Scju $=$ Scytonema julianum, Stam $=$ Stichococcus ampulliformis, Syae $=$ Synechococcus aeruginosus, Syaq $=$ Synechocystis aquatilis, Asto $=$ Astasia torta, Eugy $=$ Eutreptiella gymnastica, Eusp = Euglena spirogyra, Phon = Phacus onyx, Phor = Phacus orbicularis, Phsp = Phacus sp., Sctr $=$ Scrippsiella trochoidea, Trhi $=$ Trachelomonas hispid . 


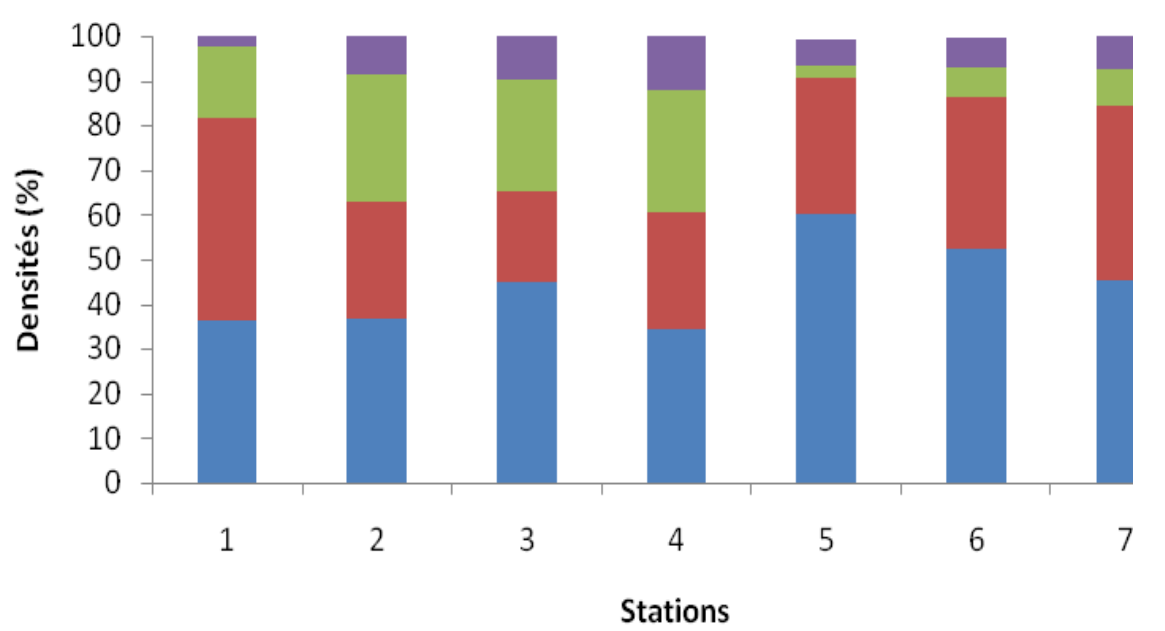

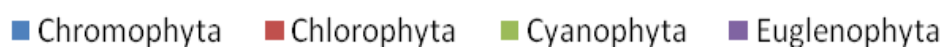

Figure 10 : Variation des densités des différents phyllums des stations des sites.

\section{DISCUSSION}

Les valeurs des différentes variables abiotiques observées montrent que les cours d'eaux considérés ont approximativement les mêmes caractéristiques physico-chimiques, malgré quelques variations observées d'une station à l'autre dans le même site mais diffèrent d'un site à un autre. Cette dissemblance dans les valeurs des paramètres physico-chimiques peut être liée aux caractéristiques des ruisseaux en amont. Car, reçoivent des polluants différents : au site 1 , les polluants proviennent des activités agropastorales alors qu'au site 2 , les polluants proviennent de la zone industrielle. Une autre possibilité qui expliquerait cela pourrait être la différence entre les substrats géoglogiques drainés par ces deux rivières; au site 1 , le substrat est vaseux tandis qu'au site 2 , il est sableux.

Etant un maillon indispensable de la chaîne trophique, les algues jouent un rôle de base dans les écosystèmes aquatiques. Par ailleurs, la composition spécifique et la dynamique des populations algales (Groga,
2012) sont influencées par d'innombrables facteurs chimiques tels que la quantité d'éléments nutritifs bio-disponibles, physiques comme la lumière, la température, l'hydrodynamique et le brassage de la colonne d'eau et par les interactions biologiques, comme le broutage par le zooplancton, la compétition interspécifique et le parasitisme. Les eaux de Kambo et Longmayagui restent chaudes. Les eaux de surface de la zone côtière du Cameroun sont chaudes toute l'année, contrairement aux eaux côtières d'autres pays comme ceux de l'Afrique de l'Ouest (Ghana, Togo, Bénin) caractérisées par un upwelling saisonnier. Les températures de surface restent toujours supérieures à $24{ }^{\circ} \mathrm{C}$ (Crosnier, 1964). La température est aussi un facteur environnemental important pour la vie aquatique contrôlant ainsi l'ensemble des processus biologiques (reproduction, croissance, préférendum thermique) liés à un environnement donné (Aminot, 1983). Elle fixe aussi la valeur de saturation des gaz dissous et notamment de l'oxygène dissous. 
La température des eaux de surface est influencée directement par les conditions climatiques (Groga, 2012). Selon Sanaa (2006), les lagunes sont soumises à des variations saisonnières de température plus importantes qu'en milieu marin. La plupart des espèces présentes dans les milieux lagunaires supportent donc une large gamme de température.

Dans un plan d'eau, la transparence varie en fonction de l'abondance des particules en suspension (argile, limon et autres particules) et $\mathrm{du}$ phytoplancton (Balvay, 1985). Les valeurs relativement faibles peuvent être attribuées à l'action du courant de l'eau, principalement. Ce dernier provoque des turbulences qui remettent en suspension les particules du sédiment (Groga, 2012). Les valeurs de la transparence dans les sites sont relativement faibles à cause de la faible profondeur de l'eau et du débit qui limitent la prolifération du phytoplancton. La profondeur et le débit jouent aussi de manière importance dans le développement algale. Dans les milieux aquatiques, l'oxygène est le moteur essentiel à la vie des organismes, il assure l'oxydation de la matière organique en faisant intervenir les différents groupements bactériens et en contribuant donc à l'autoépuration du milieu. L'oxygène est généré souvent par les algues et les végétaux aquatiques dont l'activité photosynthétique assure l'approvisionnement des organismes aquatiques en oxygène nécessaire à leur respiration, il est apporté également au milieu par ré-aération à partir de l'atmosphère ou par diffusion à partir d'eaux plus oxygénées. L'oxygène dissout est étroitement lié à la température qui conditionne sa solubilité (Sanaa, 2006). Les apports continentaux étant véhiculés par des eaux douces, la salinité paraît être leur meilleur marqueur dans les estuaires (Sanaa, 2006). Les cours d'eaux Kambo et Longmayagui restent oxygénés grâce au courant qui assure le brassage de l'eau.

Les Diatomées $(40,59 \%)$ constituent la majorité des espèces rencontrées dans les échantillons. Ces résultats sont en accord avec ceux de Konan et al. (2013) qui montrent que les Diatomées constituent $75,7 \%$ des taxons récoltés dans les rivières du Sud-Est de la côte d'Ivoire et rassemblent l'essentiel des espèces constamment rencontrées dans les échantillons. Cette dominance des Diatomées dans la composition taxonomique a été également observée par Ouattara (2000) dans la rivière Agnéby et dans les zones fluviatiles de la rivière Bia ainsi que par Round (1993) dans des cours d'eau européens. Les Diatomées sont des organismes autotrophes les plus diversifiés dans les rivières puisqu'elles ont la possibilité de coloniser toutes les surfaces disponibles. Cette facilité de colonisation justifierait leur prédominance dans la composition taxonomique des algues fixées sur feuilles. En outre, les Diatomées peuvent aussi se détacher des supports et se retrouver en dérive dans la colonne d'eau, ce qui expliquerait également leur diversité importante aussi bien dans le périphyton que dans le peuplement de pleine eau (Konan et al., 2013).

L'indice de diversité est élevé et montre que la population n'est pas soumise à l'influence d'une seule espèce qui se développe mais plutôt à un fort développement de plusieurs individus différents. D'après Kemka et al. (2004), une diversité faible caractérise une population jeune à haut pouvoir de multiplication avec prédominance d'une espèce ou d'un petit nombre d'espèces, tandis qu'une diversité élevée caractérise au contraire des populations sénescentes présentant une composition spécifique complexe. D'après les résultats obtenus, il y a une forte similitude entre la régularité et l'indice de diversité. Or Kemka et 
al. (2004) ayant fait ce constat au lac municipal de Yaoundé, signalent que la similitude entre les variations de la régularité et celles de l'indice de diversité spécifique est une preuve que ce lac est plus influencé par la richesse spécifique que par une répartition plus ou moins équitable des espèces présentes instantanément dans ce milieu.

Au site 1 , il y a une forte similitude entre les stations 2 et 3 . Ces sites présentent presque les mêmes caractéristiques et sont très peu influencés par les activités anthropiques. La station 1 est une eau marnante car il y a une rupture de communication entre elle et la station 2 par un remblaie de terre au niveau du pont de Yassa. Les algues de cette station appartiennent au périphyton car le lit de l'eau est recouvert d'une prolifération d'Eicchornia crassipes. La station 4 est soumise à une influence des activités anthropiques et diffère des autres par la couleur de l'eau qui passe de la couleur claire dans les stations 2 et 3 au vert peu foncé. Ce changement de couleur est dû à la forte présente des Chlorophycées dans le milieu. Au site 2, il y a une forte similarité entre toutes les stations qui sont beaucoup influencées par les activités anthropiques de l'amont à l'aval.

\section{Conclusion}

La présente étude a permis de connaître la composition, la structure et la dynamique spatio-temporelle des algues planctoniques et périphytiques en relation avec les caractéristiques abiotiques des rivières Kambo et Longmayagui de Douala. Les variations des paramètres abiotiques sont plus marquées au niveau spatial que temporel. A l'exception de la température, tous les paramètres mesurés varient significativement d'une rivière à une autre. En outre, la nature du substrat et des polluants influencent la conductivité et le $\mathrm{pH}$. Selon le type de cours d'eau, le débit est proportionnel à l'oxygénation et inversement proportionnel à la transparence. L'inventaire de la microflore algale a permis de recenser 105 espèces : 41 taxons de Diatomées, 29 taxons de Chlorophytes, 8 taxons d'Euglénophytes, 27 taxons de Cyanophytes. Les Bacillariophycées constituent la composante la plus importante $(40,59 \%)$ de la flore algale dans les rivières étudiées. Les compositions algales des deux cours d'eau ont montré de nombreuses similitudes du fait de leur température et $\mathrm{pH}$ assez semblables. Le nombre de taxons recensés varie de 92 (rivière Kambo) à 35 (rivière Longmayagui). Les communautés algales des stations en amont comparées à celles obtenues dans les stations en aval des cours d'eau ont montré peu de différences tant au niveau phytoplanctonique que périphytique. Le peu d'espèces qui les différencie ne permet pas de mettre en évidence une variation suivant le gradient amont-aval. Les communautés algales de la rivière Kambo comparées à celles de la rivière Longmayagui ont montré des différences. Les résultats de cette étude indiquent que la rivière Kambo est non polluée alors que la rivière Longmayagui est polluée. Une surveillance, basée sur les indices biologiques des algues pourrait être mise au point dans le but de prévenir les risques de perturbation du fait du mode de pollution en amont des cours d'eau.

\section{REFERENCES}

Aminot A. 1983. Manuel des analyses chimiques en milieu marin. Caractéristiques physicochimiques. Centre National de l'exploitation des océans ; 39-75.

Aurousseau P. 2013. Evaluation de l'impact des cours d'eau sur l'eutrophisation dans la bande côtière: nécessité de raisonner sur les concentrations et sur les flux. Biotechnol. Agron. Soc. Environ., 17(1): 271-276. 
Balvay G. 1985. Structure et Fonctionnement du Réseau Trophique dans les Retenues Artificielles. Gestion Piscicole des Lacs et Retenues Artificielles. INRA: Paris; 39-66.

Bartram J, Carmichael WW, Chorus I, Jones J, Skulberg OM. 1999. Introduction. In Toxic Cyanobacteria in Water. A Guide to their Public Health Consequences, Monitoring and Management. Chours I, Bartram J (eds). WHO: New York.

Bengtsson J. 1998. Which species? What kind of diversity? Which ecosystem function? Some problems in studies of relations between biodiversity and ecosystem function. Applied Soil Ecology, 10(3): 191-199.

Berne. 2007. Méthodes d'analyse et d'appréciation des cours d'eau. Diatomées niveau R (Région). OFEV, p. 122.

Bourrelly P. 1966. Les algues d'Eau Douce: Algues Vertes. Boubée et Cie: Paris; 511.

Bourrelly P. 1968. Les Algues d'Eau Douce: Algues Jaunes et Brunes. Boubée et Cie : Paris ; 438.

Bourrelly P. 1970. Les Algues d'Eau Douce: Algues Bleues et Rouges. Boubée et Cie : Paris ; 512.

Compère P. 1967. Algues du Sahara et de la région du Lac Tchad. Bull. Jard. Bot. Nat. Belgique, 37(2): 109-288.

Compère P. 1974. Algues de la région du lac Tchad. II Cyanophycées. Cah. O.R.S.T.O.M., Ser. Hydrobiol., 8(3-4): 165-198.

Compère P. 1976. Algues de la région du lac Tchad. VI. Chlorophycophytes $\left(2^{\mathrm{e}}\right.$ partie : Ulotrichophycées, Zygnématacées). Cah. O.R.S.T.O.M., Ser. Hydrobiol., 10(3): 135-200.

Crosnier. 1964. Fonds de pêche le long des côtes de la République Fédérale du
Cameroun. Cah. ORSTOM, $\mathrm{N}^{\mathrm{o}}$ spécial : 133.

Gaillard JF. 1993. Limnologie chimique : principes et processus. In Limnologie Générale. Edition Masson: Paris; 115156.

Groga N. 2012. Structure, fonctionnement et dynamique du phytoplancton dans le lac de Taabo (Côte d'Ivoire). Thèse de Doctorat, Université de Toulouse, p. 224.

Grönblad R, Prowse GA, Scott AM. 1958. Sudanese Desmids. Acta Botanica Fennica, 58: 1-82.

Hatcher BG, Johannes RE, Robertson AI. 1989. Review of research relevant to the conservation of shallow tropical marine ecosystems. Oceanogr. Mar. Biol. Annu. Rev., 27: 237- 414.

Kemka N, Njine T, Zébazé TSH, Niyitegeta D, Nola M, Menbohan F. 2004. Phytoplancton du lac municipal de Yaoundé: succession écologique et structure des peuplements. Journal Water Science, 17(3): 301-316.

Konan KF, Niamien-Ebrottié JE, Edia OE, Ouattara A, Gourène G. 2013. Composition et variation spatiosaisonnière du peuplement algal des rivières côtières la Cote d'Ivoire. Journal of Applied Biosciences, 66: 5147-5161.

Krammer K, Lange-Bertalot H. 2000. Bacillariophyceae. In Susswasserflora von Mitteleuropa, Ettl H, Gerloff J, Heynig H, Mollenhauer D (eds) Spektrum Akademischer Verlag: Heidelberg, Berlin; 1-5.

Li WKW. 1997. Cytometric diversity in marine ultraphytoplankton. Limnol. Oceanogr., 42(5): 874-880.

Meva'a AD, Fouda M, Bonglam CZ, Kamwo M. 2010. Analyse spatiale du risque d'inondation dans le bassin versant du Mbanya à Douala, capitale économique 
du Cameroun. Rapport, NOVATECH, Université de Douala, p. 10.

Ouattara A. 2000. Premières données systématiques et écologiques du phytoplancton du lac d'Ayamé (Côte d'Ivoire). Thèse de l'Université Catholique Leuven, Belgique, p. 200.

Round FE. 1993. A Review and Methods for the Use of Epilithic Diatoms for Detecting and Monitoring Changes in River Water Quality. Methods for the Examination of Waters and Associated Materials. HMSO Books: London; 65.

Sanaa B. 2006. Structure, dynamique et typologies phytoplanctoniques de l'estuaire du Bou Regreg (côte atlantique marocaine). Université Mohammed V - Agdal, Faculté des sciences, p. 233.

Smetaceck V, Bathmann U, Nöthing EM, Scharck R. 1991. Coastal eutrophisation: causes and consequences. In Ocean Margin Processes in Global Change, Mantoura RFC, Montin JM, Wollat R (eds). Wiley \& Sons: Chichester; 251 279.

Travers M. 1964. Diversité du microplancton du Golf de Marseille. Station marine d'Endoume et centre d'océanographie, Marseille, p. 35. 